MATHEMATICS OF COMPUTATION

Volume 73, Number 247, Pages 1547-1557

S 0025-5718(03)01612-0

Article electronically published on July 31, 2003

\title{
ORTHOMORPHISMS AND THE CONSTRUCTION OF PROJECTIVE PLANES
}

\author{
FELIX LAZEBNIK AND ANDREW THOMASON
}

\begin{abstract}
We discuss a simple computational method for the construction of finite projective planes. The planes so constructed all possess a special group of automorphisms which we call the group of translations, but they are not always translation planes. Of the four planes of order 9, three admit the additive group of the field $G F(9)$ as a group of translations, and the present construction yields all three. The known planes of order 16 comprise four selfdual planes and eighteen other planes (nine dual pairs); of these, the method gives three of the four self-dual planes and six of the nine dual pairs, including the "sporadic" (not translation) plane of Mathon.
\end{abstract}

\section{INTRODUCTION}

We discuss a simple computational method for the construction of finite projective planes. The planes so constructed all possess a special group of automorphisms which we later will define as translations, but they are not all what finite geometers call translation planes(e.g., see [3] or [10] or [12]).

Of the four planes of order 9, three admit the additive group of the field $G F(9)$ as a group of translations, and the present construction yields all three. The known planes of order 16 (see [20]) comprise four self-dual planes and eighteen other planes (nine dual pairs); of these, the method gives three of the four self-dual planes and six of the nine dual pairs, including the "sporadic" (not translation) plane of Mathon [5], [14].

\section{Graphs without 4-CyCles, Planes AND orthomorphisms}

We examine here a simple construction for graphs that are the point-line incidence graphs of projective planes. Let $G$ be a finite abelian group whose order is denoted $q$. The construction associates with each function $f: G \times G \rightarrow G$ a graph which, under certain circumstances, gives rise to a plane. It is convenient to think of $f$ as a $q \times q$ square array or matrix, so that we can speak of the rows and columns of $f$ (meaning the restrictions of $f$ to $\{a\} \times G$ or $G \times\{a\}$ for some $a \in G$ ).

2.1. Graph and plane constructions. Let $P$ and $L$ be two copies of $G \times G$. The elements of $P$ are labelled $p=\left(p_{1}, p_{2}\right)$ and are called points, while those of $L$ are labelled $l=\left[l_{1}, l_{2}\right]$ and are called lines.

Received by the editor March 13, 2002 and, in revised form, January 22, 2003.

2000 Mathematics Subject Classification. Primary 05B15, 05C50, 05C62, 51E15, 68R10.

Key words and phrases. Orthomorphisms, projective planes, translation.

This research was supported partially by a grant from the London Mathematical Society.

(C)2003 American Mathematical Society 
Given $f: G \times G \rightarrow G$, we define the bipartite graph $\Gamma_{f}$ in the following way. The vertex set of $\Gamma_{f}$ is $P \cup L, P$ and $L$ are color classes, and for $p=\left(p_{1}, p_{2}\right) \in P$, $l=\left[l_{1}, l_{2}\right] \in L$,

$$
p l \in E\left(\Gamma_{f}\right) \text { iff } p_{2}+l_{2}=f\left(p_{1}, l_{1}\right) .
$$

Clearly the graphs $\Gamma_{f}$ having order $2 q^{2}$ are $q$-regular; indeed, each point $p \in P$ has, for each $l_{1} \in G$, exactly one neighbour $\left[l_{1}, l_{2}\right]$, and likewise each line $l \in L$ has exactly one neighbour $\left(p_{1}, p_{2}\right)$ for each $p_{1} \in G$. Hence the size (number of edges) of $\Gamma_{f}$ is $q^{3}$.

Next we define a bipartite graph $\Pi_{f}$ which contains $\Gamma_{f}$ as an induced subgraph. Let $P^{*}$ be the set formed from $P$ by adding the $q+1$ elements $(p)$, where $p \in G$, and $(\infty)$. Likewise let $L^{*}$ be formed by adding to $L$ the $q+1$ elements $[l], l \in G$, and $[\infty]$. The bipartite graph $\Pi_{f}$ has vertex set $P^{*} \cup L^{*}$; it contains the graph $\Gamma_{f}$ as an induced subgraph, together with additional edges

$$
\begin{gathered}
\left\{(p)\left[p, l_{2}\right]: p, l_{2} \in G\right\}, \\
\left.\{l]\left(l, p_{2}\right): l, p_{2} \in G\right\}, \\
\{(\infty)[l]: l \in G\}, \\
\{(p)[\infty]: p \in G\},
\end{gathered}
$$

and $(\infty)[\infty]$. Therefore graph $\Pi_{f}$ has both color classes of cardinality $q^{2}+q+1$ and is regular of degree $q+1$. It also follows from the definition of $\Pi_{f}$ that it contains no 4-cycle if and only if graph $\Gamma_{f}$ contains no 4-cycles. The following statement is an immediate corollary of Bollobás [1, Lemma 2.1, pages 309-310] and can be easily checked.

Proposition. Graph $\Pi_{f}$ is isomorphic to the point-line incidence graph of a projective plane of order $q$ if and only if $\Gamma_{f}$ contains no 4-cycles.

(The above method of extending a graph $\Gamma_{g}$ to the point-line incidence graph was shown to one of the authors by Ustimenko [21.) Now two distinct vertices $\left(p_{1}, p_{2}\right)$ and $\left(p_{1}^{\prime}, p_{2}^{\prime}\right)$ of the graph $\Gamma_{f}$ have a common neighbour $\left[l_{1}, l_{2}\right]$ if and only if $p_{2}-p_{2}^{\prime}=f\left(p_{1}, l_{1}\right)-f\left(p_{1}^{\prime}, l_{1}\right)$ (and so necessarily $p_{1} \neq p_{1}^{\prime}$ ). Hence these vertices lie in a 4-cycle if and only if there is some $l_{1}^{\prime} \neq l_{1}$ such that $f\left(p_{1}, l_{1}\right)-f\left(p_{1}^{\prime}, l_{1}\right)=$ $f\left(p_{1}, l_{1}^{\prime}\right)-f\left(p_{1}^{\prime}, l_{1}^{\prime}\right)$. So the condition that $\Gamma_{f}$ have no 4 -cycles is equivalent to the following simple condition on the function $f$ :

$$
f(a, b)-f\left(a^{\prime}, b\right) \neq f\left(a, b^{\prime}\right)-f\left(a^{\prime}, b^{\prime}\right) \quad \text { for all } a \neq a^{\prime}, b \neq b^{\prime} \in G
$$

or, equivalently,

$$
f(a, b)-f\left(a, b^{\prime}\right) \neq f\left(a^{\prime}, b\right)-f\left(a^{\prime}, b^{\prime}\right) \quad \text { for all } a \neq a^{\prime}, b \neq b^{\prime} \in G .
$$

We call the function $d: G \rightarrow G$ given by $d(b)=f(a, b)-f\left(a^{\prime}, b\right)$ the difference of the two rows of $f$ indexed by $a$ and $a^{\prime}$. Thus, $\Gamma_{f}$ has no 4-cycles if and only if every two rows of $f$ differ by a permutation of $G$, or, equivalently, every two columns differ by a permutation of $G$. We call a function $f$ satisfying this condition a plane function, since $\Pi_{f}$ is a projective plane if and only if $f$ is a plane function.

Remark. Our motivation for these definitions was to study the most general functions $f$ that would produce graphs of girth 4 subject to the condition $(\dagger)$. The condition $(\dagger)$ itself is a special case of a general construction discussed by Lazebnik and Woldar [13], which was in turn motivated by the construction of graphs of large 
girth. The number of all functions $f: G \times G \rightarrow G$ is very large when $|G|=16$ but, using the observations developed below, it is possible to make an exhaustive analysis of the case when $G$ is the additive group of the field $\mathbb{F}_{16}=G F(16)$.

Remark. Our notion of a plane function should not be confused with the notion of a planar function introduced by Dembowski and Ostrom in [4] and later used extensively in the contexts of both projective planes and permutation polynomials (see, e.g., Rónyai and Szőnyi [18, Hiramine [11], Gluck [9], Coulter and Matthews [2]). Given two finite groups $G$ and $H$ of the same order, both written additively, but not necessarily commutative, a function $g: G \rightarrow H$ is called planar if for every $a \in G \backslash\{0\}$ the functions defined by $x \rightarrow g(a+x)-g(x)$ and $x \rightarrow-g(x)+g(x+a)$ are both bijections from $G$ to $H$. In the case when both $G$ and $H$ are additive groups of the field $\mathbb{F}_{q}$, planar functions are often referred to as difference permutation polynomials. In this case, following [4, given a planar function $g$, an incident structure leading to a part of an affine plane is defined as follows: "Points" are the elements of $\mathbb{F}_{q} \times \mathbb{F}_{q}$. "Lines" are the symbols $L(a, b)$, with $(a, b) \in \mathbb{F}_{q} \times \mathbb{F}_{q}$, and the "incidence" I is defined by

$$
(x, y) \mathrm{I} L(a, b) \quad \text { if and only if } y=g(x-a)+b .
$$

So a planar function $g$ is equivalent (subject to the unimportant replacement of $(a, b)$ by $(-a,-b))$ to a plane function of the special form $f(x, y)=g(x+y)$. However, in the case when $G$ is the additive group of a field of characteristic 2 , no functions $f$ of this kind can satisfy the criterion to be plane, since if $a=b^{\prime} \neq a^{\prime}=b$, then $f(a, b)-f\left(a^{\prime}, b\right)=g(a+b)+g(0)=f\left(a, b^{\prime}\right)-f\left(a^{\prime}, b^{\prime}\right)$. The main results of this paper correspond to the characteristic 2 case, so unfortunately the theory of planar functions cannot help.

2.2. Isomorphisms. Let $f, g: G \times G \rightarrow G$ and suppose that $\phi: \Gamma_{f} \rightarrow \Gamma_{g}$ is a graph isomorphism. If $\phi$ has the property that $\phi: P \rightarrow P, \phi: L \rightarrow L$, and the first coordinates of $\phi(p)$ and $\phi(l)$ depend only on the first coordinates of $p$ and $l$, respectively, then $\phi$ extends naturally to an isomorphism $\phi^{*}: \Pi_{f} \rightarrow \Pi_{g}$. In this case, we call $\phi$ a plane isomorphism, since if $\Gamma_{f}$ contains no 4 -cycles, then $\phi^{*}$ is an isomorphism of projective planes.

Here are a few straightforward such isomorphisms.

(1) Translate. Given $a \in G$, let $\phi: \Gamma_{f} \rightarrow \Gamma_{f}$ be given by

$$
\left(p_{1}, p_{2}\right) \mapsto\left(p_{1}, p_{2}+a\right) \quad \text { and } \quad\left[l_{1}, l_{2}\right] \mapsto\left[l_{1}, l_{2}-a\right]
$$

For every function $f$, this gives a plane automorphism of $\Gamma_{f}$ to itself. We call the corresponding automorphism of the projective plane a translation. Thus, all the planes we construct admit a group of translation which is isomorphic to $G$, though they are not all translation planes in the sense of [10 or 3 or 12, whose definition we decide to omit.

(2) Zeroize. Let $c, d: G \rightarrow G$ and let $f, g: G \times G \rightarrow G$ be given by

$$
g(x, y)=f(x, y)+c(x)+d(y) .
$$

Then there is a plane isomorphism $\phi: \Gamma_{f} \rightarrow \Gamma_{g}$ given by

$$
\left(p_{1}, p_{2}\right) \mapsto\left(p_{1}, p_{2}+c\left(p_{1}\right)\right) \quad \text { and } \quad\left[l_{1}, l_{2}\right] \mapsto\left[l_{1}, l_{2}+d\left(l_{1}\right)\right] .
$$

In particular, by choosing suitable $c$ and $d$, we see that every $\Gamma_{f}$ is plane isomorphic to a $\Gamma_{g}$ where the first row and column of $g$ are zero; that is, $g(0, a)=g(a, 0)=0$ for all $a \in G$. 
(3) Permute. Let $\sigma$ and $\tau$ be permutations of $G$, and let $f, g: G \times G \rightarrow G$ be given by

$$
g(x, y)=f(\sigma x, \tau y) .
$$

Then there is a plane isomorphism $\phi: \Gamma_{f} \rightarrow \Gamma_{g}$ given by

$$
\left(p_{1}, p_{2}\right) \mapsto\left(\sigma^{-1} p_{1}, p_{2}\right) \quad \text { and } \quad\left[l_{1}, l_{2}\right] \mapsto\left[\tau^{-1} l_{1}, l_{2}\right]
$$

In particular, by choosing suitable $\sigma$ and $\tau$, we see that every $\Gamma_{f}$ for which $f$ is a plane function is plane isomorphic to a $\Gamma_{g}$ where the second row and column of $g$ are the identity map; that is, $g(1, a)=g(a, 1)=a$ for all $a \in G$, where 1 denotes the second element in some ordering of $G$ (the first element being 0 ).

Note that this operation applied to a zeroized map (as just previously described) produces another zeroized map. That is, every such $\Gamma_{f}$ is plane isomorphic to a $\Gamma_{g}$ where the first row and column of $g$ are zero and the second row and column are the identity. We call such a $g$ normalized.

(4) Transpose. Let $f, g: G \times G \rightarrow G$ be given by

$$
g(x, y)=f(y, x) .
$$

Then there is an isomorphism $\phi: \Gamma_{f} \rightarrow \Gamma_{g}$ given by

$$
\left(p_{1}, p_{2}\right) \mapsto\left[p_{1}, p_{2}\right] \quad \text { and } \quad\left[l_{1}, l_{2}\right] \mapsto\left(l_{1}, l_{2}\right) .
$$

This isomorphism is not a plane isomorphism, but there is a natural isomorphism between the plane $\Pi_{f}$ and the dual of the plane $\Pi_{g}$. Note that, if $f$ is normalized (in the sense just previously described), then so is $g$.

(5) Additive transform. Let $A: G \rightarrow G$ be an isomorphism of group $G$ : that is, $A$ is a bijection and $A(a+b)=A(a)+A(b)$ for all $a, b \in G$. Let $f, g: G \times G \rightarrow G$ be given by

$$
g(x, y)=A f(x, y) .
$$

Then there is a plane isomorphism $\phi: \Gamma_{f} \rightarrow \Gamma_{g}$ given by

$$
\left(p_{1}, p_{2}\right) \mapsto\left(p_{1}, A p_{2}\right) \quad \text { and } \quad\left[l_{1}, l_{2}\right] \mapsto\left[l_{1}, A l_{2}\right] \text {. }
$$

The use of the isomorphisms (1) - (5) reduces the number of functions $f$ that need to be examined in any exhaustive computational search.

2.3. Orthomorphisms. As shown above, we may restrict our attention to normalized functions. A normalized plane function has its first row and column zero, its second row and column are the identity, and each row differs from any other by a permutation.

An orthomorphism of $G$ is a permutation $\sigma$ of $G$, such that $\sigma$ differs from the identity by a permutation; that is, the map $a \mapsto \sigma a-a$ is a permutation of $G$.

Therefore $f$ is a normalized plane function if and only if its first row and column are zero, its second row and column are the identity, and all other rows are orthomorphisms which fix zero and which pairwise differ by a permutation (or, equivalently, all other columns are orthomorphisms which pairwise differ by a permutation). 
For example, if $G$ is the field of order 5 and $f: G \times G$ is the function described by the array

$\begin{array}{lllll}0 & 0 & 0 & 0 & 0 \\ 0 & 1 & 2 & 3 & 4 \\ 0 & 2 & 4 & 1 & 3 \\ 0 & 3 & 1 & 4 & 2 \\ 0 & 4 & 3 & 2 & 1\end{array}$

then $f$ is normalized; moreover, each row is an orthomorphism (for instance, the fourth row differs from the identity by the permutation 02413), and the rows differ pairwise by permutations. Thus $f$ is a normalized plane function and so $\Pi_{f}$ is a projective plane - namely, of course, the Desarguesian plane of order 5.

The existence of orthomorphisms for a finite abelian group is given by a theorem of Paige [16, 17]: a finite abelian group admits an orthomorphism if and only if its Sylow 2-subgroup is trivial or non-cyclic. A proof can also be found in Evans 7, page 19]. The necessity of the conditions is also proved in van Lint and Wilson [22, page 264]. It is easy to see that if a group $G$ has an odd order, then the map $g \rightarrow g^{2}$ is an orthomorphism of $G$. Paige's theorem implies that $\mathbb{Z}_{n}$ admits orthomorphisms only if $n$ is odd. For much more on orthomorphisms, on their relation to other notions of combinatorics and geometry, and on orthomorphism graphs (next section) one can consult an excellent monograph by Evans [7].

\section{THE ORTHOMORPHISM GRAPH}

We consider only orthomorphisms that fix zero; note that, if $\pi$ is any orthomorphism, then so is the map $x \mapsto \pi(x+a)+b$ for any two elements $a, b \in G$, and it is easy to choose $a$ and $b$ so that the resulting orthomorphism fixes zero.

The orthomorphism graph $\operatorname{Orth}(G)$ has, as vertices, all orthomorphisms of $G$ that fix zero, and $\pi \sigma$ is an edge of $\operatorname{Orth}(G)$ if $\pi-\sigma$ is an orthomorphism of $G$ (necessarily fixing zero).

Let $a$ be any non-zero element of $G$ and let $\pi$ be a vertex of $\operatorname{Orth}(G)$. Then $\pi(a) \neq 0$ since $\pi$ fixes zero, and $\pi(a) \neq a$ since $\pi$ minus the identity is a permutation that fixes zero. Thus $\pi(a) \in G-\{0, a\}$. Moreover, if $\sigma$ is another vertex of $G$ and $\pi(a)=\sigma(a)$, then $(\pi-\sigma)(a)=0=(\pi-\sigma)(0)$ so $\pi \sigma$ is not an edge of $G$. Therefore $\operatorname{Orth}(G)$ is a $(|G|-2)$-partite graph.

It follows from the definition of $\operatorname{Orth}(G)$ and from the description of a normalized plane function given in the previous section that normalized plane functions correspond exactly to complete subgraphs, or cliques, of order $|G|-2$ in $\operatorname{Orth}(G)$. Together with a zero row and the identity row, the orthomorphisms forming the vertices of a clique of order $|G|-2$ furnish the rows of a normalized plane function.

In the case that $G=\mathbb{F}_{q}$, the finite field of order $q$, there is always at least one clique, namely the "trivial" or "linear" clique, whose vertices are the orthomorphisms $x \mapsto a x$, for each $a \in \mathbb{F}_{q}-\{0,1\}$. The normalized plane functions corresponding to these linear cliques produce the Desarguesian planes of order $q$.

More generally, if $q=p^{k}$ where $p$ is prime, and if $A: \mathbb{F}_{q} \rightarrow \mathbb{F}_{q}$ is an $\mathbb{F}_{p}$-linear map, then the map $x \mapsto A x$ is an orthomorphism provided that both $A$ and $A-I$ are non-singular. If the eigenvalues of $A$ are all primitive roots of $\mathbb{F}_{q}$, then $A^{d}$ is an orthomorphism for every $d \leq q-2$. In this case, the maps $x \mapsto A^{d} x, 1 \leq d \leq q-2$, form a $(q-2)$-clique in $\operatorname{Orth}\left(\mathbb{F}_{q}\right)$. The plane resulting from the corresponding normalized plane function is Desarguesian. Examples of other cliques in $\operatorname{Orth}\left(\mathbb{F}_{q}\right)$ 
can be found in Evans [7] or in Wan, Mullen and Shiue [24]. It is a long-standing conjecture that non-Desarguesian planes of prime order do not exist. A weaker long standing conjecture is that non-Desarguesian planes of prime order which admit translations do not exist. In this context the use of the term "translation" is distinct from its regular use in finite geometries. Let $p$ be a point of a projective plane $\pi$ and let $l$ be a line through $p$. A $(p, l)$-elation of $\pi$ is a collineation of $\pi$ which fixes (as a set) every line through $p$ and every point on $l$. If $l=l_{\infty}$, the elation induces a collineation of the corresponding affine plane which is called a translation of the affine plane. When we say that $\pi$ admits a translation, we really mean that $\pi$ admits a $(p, l)$-elation. This is consistent with the definition of translation given in subsection 2.2. The relation between the second conjecture and maximum cliques in $\operatorname{Orth}\left(\mathbb{F}_{p}\right)$ is given by a theorem by Evans and McFarland [8], which states that a non-Desarguesian plane of prime order, admitting translations, exists if and only if $\operatorname{Orth}\left(\mathbb{F}_{p}\right)$ contains more than one $(p-2)$-clique.

Much more on this relation, including proofs, can be found in Evans 7 .

\section{Computational Results}

The orthomorphism graphs $\operatorname{Orth}\left(\mathbb{F}_{q}\right)$, where $q \leq 16$ is a prime power, were constructed by computer, and the $(q-2)$-cliques in the graph were found. The results are summarized in Table 1. In this table, the column headed $n$ shows the

TABLE 1. Properties of the orthomorphism graphs $\operatorname{Orth}\left(\mathbb{F}_{q}\right)$.

\begin{tabular}{|r|ccccc|}
\hline$q$ & $n$ & $e$ & core & $\max 2$ & $K_{q-2}$ 's \\
\hline 3 & 1 & 0 & 1 & 0 & 1 \\
4 & 2 & 1 & 2 & 0 & 1 \\
5 & 3 & 3 & 3 & 0 & 1 \\
7 & 19 & 10 & 5 & 1 & 1 \\
8 & 48 & 288 & 48 & 2 & 8 \\
9 & 249 & 1248 & 249 & 4 & 21 \\
11 & 3441 & 2016 & 141 & 3 & 1 \\
13 & 79259 & 395242 & 271 & 5 & 1 \\
16 & 15296512 & 2199658496 & - & - & 2439392 \\
\hline
\end{tabular}

number of vertices in $\operatorname{Orth}\left(\mathbb{F}_{q}\right)$ and the column headed $e$ shows the number of edges. The column headed "core" shows the number of vertices in the $(q-3)$-core of $\operatorname{Orth}\left(\mathbb{F}_{q}\right)$, the $(q-3)$-core being the subgraph of minimum degree $q-3$ formed by repeatedly removing vertices of degree less than $q-3$ until none remain (of course, all $(q-2)$-cliques lie within the $(q-3)$-core). The column headed "max2" shows the number of vertices in a second-largest maximal complete subgraph, that is, a largest maximal complete subgraph with fewer than $q-2$ vertices. The final column shows the number of $(q-2)$-cliques in $\operatorname{Orth}\left(\mathbb{F}_{q}\right)$.

4.1. The graphs $\operatorname{Orth}\left(\mathbb{F}_{p}\right)$ for prime $p \leq 13$. For $q \leq 7$ the graph $\operatorname{Orth}\left(\mathbb{F}_{q}\right)$ has a very simple structure; in fact $\operatorname{Orth}\left(\mathbb{F}_{q}\right)=K_{q-2}$ for $q \leq 5$, and $\operatorname{Orth}\left(\mathbb{F}_{7}\right)$ consists of $K_{5}$ together with 14 isolated vertices. The graph $\operatorname{Orth}\left(\mathbb{F}_{11}\right)$ has 2640 isolated vertices, and all other vertices have degree at most 8 except for six vertices of degree 162; these vertices correspond to the orthomorphisms $x \mapsto a x$ for $a \in$ 
$\{3,4,5,7,8,9\}$. The graph $\operatorname{Orth}\left(\mathbb{F}_{13}\right)$ is relatively more dense; it has only 260 isolated vertices, and 69639 of the 79259 vertices are in the 6 -core. But the 7-core has only 973 vertices.

The graphs $\operatorname{Orth}\left(\mathbb{F}_{p}\right)$ for $p$ an odd prime appear to have the property that the second largest clique has $(p-5) / 2$ vertices for $p \equiv 3(\bmod 4)$ and $(p-3) / 2$ vertices for $p \equiv 1(\bmod 4), p>5$. Evans [6] constructed maximal cliques of these sizes in $\operatorname{Orth}\left(\mathbb{F}_{p}\right)$.

4.2. The graphs $\operatorname{Orth}\left(\mathbb{F}_{q}\right)$ for $q=8,9$. The graph $\operatorname{Orth}\left(\mathbb{F}_{8}\right)$ is 12-regular. The normalized plane functions corresponding to the eight 6-cliques are isomorphic to each other under the isomorphisms described in subsection 2.2, each therefore giving rise to the Desarguesian plane.

The graph $\operatorname{Orth}\left(\mathbb{F}_{9}\right)$ has minimum degree 6 . It has twenty-one $K_{7}$ 's, falling into three orbits under the isomorphisms of subsection 2.2 these orbits correspond to the Desarguesian plane (three $K_{7}$ 's), another plane and its dual plane (nine $K_{7}$ 's each). These three planes are all the three planes of order 9 that admit a translation. The last two non-Desarguesian planes are members of an infinite class of so-called Hall planes; see [12. It is well known that there are exactly four projective planes of order 9, e.g., see [19]. The one that our method does not find is a member of an infinite class of so-called Hughes planes. Hughes planes are self-dual of order $q^{2}$ and their collineation groups have no subgroup isomorphic to the additive group of $G F\left(q^{2}\right)$ (follows from [12, Corollary 2, page 201] or from [3, page 247]. All planes of order 9 were discovered by Veblen and Wedderburn 23.

4.3. The graphs $\operatorname{Orth}\left(\mathbb{F}_{16}\right)$. The graph $\operatorname{Orth}\left(\mathbb{F}_{16}\right)$ has minimum degree 109; it has 112 vertices of degree 60160 but the next largest degree is 9984 , and the average degree is only 143.8 .

There are $2439392 K_{14}$ 's falling into thirty-three orbits under the isomorphisms of subsection 2.2 Unlike the case for $q<16$, it happens here that $(q-2)$-cliques which are not isomorphic under the isomorphisms of subsection 2.2 sometimes give rise to isomorphic projective planes. In fact, exactly fifteen planes appear, three being self-dual and the remainder forming six dual pairs. The planes that appear are all known. Table 2 lists each plane found, together with the number of $K_{14}$ 's giving rise to it, and the number of the thirty-three orbits that these $K_{14}$ 's make up. The first three planes are self-dual - of the remaining twelve planes, only one

TABLE 2. Planes arising from 14-cliques in $\operatorname{Orth}\left(\mathbb{F}_{16}\right)$.

\begin{tabular}{|l|cc|}
\hline Plane & $K_{14}$ 's & Orbits \\
\hline PG(2,16) & 336 & 1 \\
SEMI2 & 554400 & 3 \\
SEMI4 & 92400 & 3 \\
HALL & 22848 & 2 \\
LMRH & 81600 & 2 \\
JOWK & 81600 & 2 \\
DSFP & 380800 & 3 \\
DEMP & 228480 & 3 \\
MATH & 100800 & 1 \\
\hline
\end{tabular}


is listed for each dual pair, the numbers for a plane and its dual being the same.

The names of the planes and their descriptions are taken from Royle [20]. The three self-dual planes are the Desarguesian plane PG(2,16) together with SEMI2 and SEMI4, the semifield planes with kernels $\mathbb{F}_{2}$ and $\mathbb{F}_{4}$. The other planes are HALL - the Hall plane, LMRH - the Lorimer-Rahilly plane, JOWK - the JohnsonWalker plane, DSFP - the derived semifield plane, DEMP - the Dempwolff plane, and MATH - the Mathon plane (together with their duals).

It might be noted that one of the planes produced is not a translation plane, namely MATH. Representative normalized plane functions are given for the three self-dual planes in Table 3 and for the six non-self-dual planes in Table 4. Since only the additive structure of $\mathbb{F}_{16}$ is involved, the field can be regarded as $\mathbb{F}_{2}^{4}$ under addition, and its elements thus correspond in a natural way to the numbers $0, \ldots, 15$. These numbers are written in hexadecimal in Tables 3 and 4 .

TABLE 3. Normalized plane functions for some self-dual planes of order 16

PG(2,16)
0000000000000000
0123456789abcdef
02318ab9cefd4675
0312cfde47568b9a
048c62eabf37d951
05af278d369c14be
06bde85371ca9f24
079ead34f86152cb
08c4b37fd5196ea2
09e7f6185cb2a34d
Oaf539c61be428d7
Obd67ca1924fe538
0c48d1956a2eb7f3
0d6b94f2e3857a1c
0e795b2ca4d3f186
0f5a1e4b2d783c69

SEMI2
0000000000000000
0123456789abcdef
02318ab9cefd4675
0312cfde47568b9a
04c8519dfb37ae62
05eb14fa729c638d
06f9db2435cae817
07da9e43bc6125f8
084ce6a291d57f3b
096fa3c5187eb2d4
0a7d6c1b5f28394e
0b5e297cd683f4a1
0c84b73f6ae2d159
0da7f258e3491cb6
0eb53d86a41f972c
0f9678e12db45ac3

SEMI4 0000000000000000 $0123456789 a b c d e f$ 02318 ab9cefd 4675 0312 cfde $47568 b 9 a$ 049d51c8ea73bf 26 05be14af63d872c9 $06 \mathrm{acdb} 71248 \mathrm{ef} 953$ $078 \mathrm{f} 9 \mathrm{e} 16 \mathrm{ad} 2534 \mathrm{bc}$ $08 \mathrm{e} 6 \mathrm{a} 24 \mathrm{c} 7 \mathrm{f} 91 \mathrm{~d} 53 \mathrm{~b}$ $09 \mathrm{c} 5 \mathrm{e} 72 \mathrm{bf} 63 \mathrm{a} 18 \mathrm{~d} 4$ 0ad728f $5 \mathrm{~b} 16 \mathrm{c} 934 \mathrm{e}$ Obf $46 \mathrm{~d} 9238 \mathrm{c} 75 \mathrm{ea} 1$ $0 \mathrm{c} 7 \mathrm{bf} 38495 \mathrm{e} 26 \mathrm{a} 1 \mathrm{~d}$ $0 d 58 b 6 e 31 c 49 a 7 f 2$ $0 \mathrm{e} 4 \mathrm{a} 793 \mathrm{~d} 5 \mathrm{~b} 1 \mathrm{f} 2 \mathrm{c} 68$ 0f693c5ad2b4e187

4.4. Computational method. Programs were written in the $\mathrm{C}$ language and were run on $800 \mathrm{MHz}$ Pentium machines operating Red Hat Linux version 7.2 , at the Department of Pure Mathematics in Cambridge.

All the data for $q \leq 11$ can be computed in less than a couple of seconds. For $q=13$ the orthomorphisms were found in about three seconds but the construction and analysis of the graph $\operatorname{Orth}\left(\mathbb{F}_{13}\right)$ took a further twenty-five minutes.

For $q=16$, the list of 15296512 orthomorphisms was found in just under a day by sixty machines working in parallel. The analysis of the graph and the enumeration of the $K_{14}$ 's, along with their orbits, was performed by thirteen machines acting in parallel over a period of about four days (these machines being the available ones with the required $380 \mathrm{Mb}$ of memory).

In order to find isomorphisms between the thirty-three orbits and to find isomorphisms between the planes produced by the above method and the known planes listed on [20], the excellent nauty program of Brendan McKay [15] was used, via 
TABLE 4. Normalized plane functions for some non-self-dual planes

$\begin{array}{ccc}\text { HALL } & \text { LMRH } & \text { JOWK } \\ \text { 0000000000000000 } & \text { 0000000000000000 } & \text { 000000000000000 } \\ \text { 0123456789abcdef } & \text { 0123456789abcdef } & \text { 0123456789abcdef } \\ \text { 02318ab9cefd4675 } & \text { 02318ab9cefd4756 } & \text { 02318ab9dfec5764 } \\ \text { 0312cfde47568b9a } & \text { 0312cfde47568ab9 } & \text { 0312cfde56479a8b } \\ \text { 048c62eb5f3a79d1 } & \text { 048d2e5a916c3f7b } & \text { 049d51c8ea73bf26 } \\ \text { 05af278cd691b43e } & \text { 05ae6b3d18c7f294 } & \text { 05be14af63d872c9 } \\ \text { 06bde85291c73fa4 } & \text { 06bca4e35f91782d } & \text { 06acdb71359fe842 } \\ \text { 079ead35186cf24b } & \text { 079fe184d63ab5c2 } & \text { 078f9e16bc3425ad } \\ \text { 08c4b37da51f9e62 } & \text { 08c637afe2b419d5 } & \text { 08c4e63ba25d19f7 } \\ \text { 09e7f61a2cb4538d } & \text { 09e572c86b1fd43a } & \text { 09e7a35c2bf6d418 } \\ \text { 0af539c46be2d817 } & \text { 0af7bd162c495e83 } & \text { 0af56c827db14e93 } \\ \text { 0bd67ca3e24915f8 } & \text { 0bd4f871a5e2936c } & \text { 0bd629e5f41a837c } \\ \text { 0c48d196fa25e7b3 } & \text { 0c4b19f573d826ae } & \text { 0c59b7f3482ea6d1 } \\ \text { 0d6b94f1738e2a5c } & \text { 0d685c92fa73eb41 } & \text { 0d7af294c1856b3e } \\ \text { 0e795b2f34d8a1c6 } & \text { 0e7a934cbd2561f8 } & \text { 0e683d4a97c2f1b5 } \\ \text { 0f5a1e48bd736c29 } & \text { 0f59d62b348eac17 } & \text { 0f4b782d1e693c5a } \\ \text { DSFP } & & \\ \text { 0000000000000000 } & \text { 0000000000000000 } & \text { 000000000000000 } \\ \text { 0123456789abcdef } & \text { 0123456789abcdef } & \text { 0123456789abcdef } \\ \text { 02318ab9cefd4756 } & \text { 02318ab9cfde5764 } & \text { 02318ab9cefd6457 } \\ \text { 0312cfde47568ab9 } & \text { 0312cfde46759a8b } & \text { 0312cfde4756a9b8 } \\ \text { 04c96e1ab53fd287 } & \text { 04e96bf3581ca2d7 } & \text { 049d51c8eb72fa63 } \\ \text { 05ea2b7d3c941f68 } & \text { 05ca2e94d1b76f38 } & \text { 05be14af62d9378c } \\ \text { 06f8e4a37bc295d1 } & \text { 06d8e14a97c2f5b3 } & \text { 06acdb71349e8f25 } \\ \text { 07dba1c4f269583e } & \text { 07fba42d1e69385c } & \text { 078f9e16bd3542ca } \\ \text { 084eb72fda156c93 } & \text { 087e36cba25d49f1 } & \text { 08e6b35da14f7c92 } \\ \text { 096df24853bea17c } & \text { 095d73ac2bf6841e } & \text { 09c5f63a28e4b17d } \\ \text { 0a7f3d9614e82bc5 } & \text { 0a4fbc726d831e95 } & \text { 0ad739e45c812bf6 } \\ \text { 0b5c78f19d43e62a } & \text { 0b6cf915e428d37a } & \text { 0bf47c83d52ae619 } \\ \text { 0c87d9356f2abe14 } & \text { 0c975d38fa41eb26 } & \text { 0c7be2951f68d3a4 } \\ \text { 0da49c52e68173fb } & \text { 0db4185f73ea26c9 } & \text { 0d58a7f296c31e4b } \\ \text { 0eb6538ca1d7f942 } & \text { 0ea6d781359fbc42 } & \text { 0e4a682cf3b795d1 } \\ \text { 0f9516eb287c34ad } & \text { 0f8592e6bc3471ad } & \text { 0f692d4b7a1c583e }\end{array}$

the dreadnaut utility. This program allows the user to compute certain invariants of a graph which assist in establishing isomorphisms (or the absence of them) between graphs. Two invariants that are thought to work well with the graphs of projective planes, called cellfano and cellfano2, have been added to the library of invariants in version 2.0 of nauty; the second of these new invariants was used in the detection of isomorphisms between the planes in this study, and only about four minutes were needed on average to determine whether two planes were isomorphic (the time required being very much larger without the use of the invariant). 
4.5. Other abelian groups. We have concentrated so far on the case when $G=$ $\mathbb{F}_{q}$ but in fact it is only the additive group of the field that we have made use of, and we might just as well have used any abelian group for $G$. As mentioned in subsection 2.3 it is necessary for $G$ to have trivial or non-cyclic Sylow 2-subgroup in order for it to have any orthomorphisms at all. Writing $\mathbb{Z}_{n}$ for the cyclic group of order $n$, the groups we have discussed are $\mathbb{Z}_{p}$ for $p$ prime, together with $\mathbb{Z}_{2}^{2}, \mathbb{Z}_{2}^{3}$, $\mathbb{Z}_{3}^{2}$ and $\mathbb{Z}_{2}^{4}$, the additive groups of $\mathbb{F}_{4}, \mathbb{F}_{8}, \mathbb{F}_{9}$ and $\mathbb{F}_{16}$. Of the remaining groups of order at most $16, \mathbb{Z}_{9}$ has 225 orthomorphisms and $\mathbb{Z}_{15}$ has 2424195 . $\mathbb{Z}_{2} \times \mathbb{Z}_{4}$ has 48 orthomorphisms, and $\mathbb{Z}_{2}^{2} \times \mathbb{Z}_{3}$ has 16512 . These data confirm the results obtained by many authors and are surveyed in Chapter 6 of [7]. The following results are new: $\mathbb{Z}_{2} \times \mathbb{Z}_{8}$ has 14735360 orthomorphisms, $\mathbb{Z}_{2}^{2} \times \mathbb{Z}_{4}$ has 14886912 and $\mathbb{Z}_{4}^{2}$ has 14813184. The maximum size of a complete subgraph in the orthomorphism graph of $\mathbb{Z}_{2} \times \mathbb{Z}_{8}$ is 3 , and the graph contains $256000 K_{3}$ 's. The corresponding data for $\mathbb{Z}_{2}^{2} \times \mathbb{Z}_{4}$ and $\mathbb{Z}_{4}^{2}$ are $1062656 K_{6}$ 's and $21504 K_{6}$ 's. It would be interesting to know whether the orthomorphism graph of an abelian group of order $n$ can ever contain an $(n-2)$-clique if the group is not a power of some prime order group.

\section{Concluding Remarks}

As we stated in subsection 2.2(1), all projective planes produced by our approach will admit a group of automorphisms formed by translations and isomorphic to $G$. Therefore, in order to find new projective planes, one may try to get rid of this property by changing $p_{2}+l_{2}$ in the definition of $\Gamma_{f}$ to $g\left(p_{2}, l_{2}\right)$, where $g: G \times G \rightarrow G$ is an arbitrary function and $G$ is an abelian group of order $q$. Given $f, g: G \times G \rightarrow$ $G$, we define the bipartite graph $\Gamma_{f, g}$ in the following way. The vertex set of $\Gamma_{f, g}$ is $P \cup L, P=L=G \times G$ are color classes, and for $p=\left(p_{1}, p_{2}\right) \in P, l=\left[l_{1}, l_{2}\right] \in L$,

$$
p l \in E\left(\Gamma_{f, g}\right) \text { iff } g\left(p_{2}, l_{2}\right)=f\left(p_{1}, l_{1}\right) .
$$

The following property on $f, g$ makes $\Gamma_{f, g} q$-regular: for given $p_{1}, p_{2}$ and $l_{1}$, the equation $g\left(p_{2}, l_{2}\right)=f\left(p_{1}, l_{1}\right)$ has a unique solution for $l_{2}$, and symmetrically, for given $l_{1}, l_{2}$ and $p_{1}$, the equation $g\left(p_{2}, l_{2}\right)=f\left(p_{1}, l_{1}\right)$ has a unique solution for $p_{2}$.

Let $A=A(f)$ and $B=B(g)$ be the matrices (tables) of the functions $f$ and $g$, respectively, where rows correspond to points (or $p$ 's) and columns to lines (or $l$ 's). Proofs of the following observations are straightforward:

- $\Gamma_{f, g}$ is $q$-regular if every row and every column of $B$ contains every entry of $A$ (i.e., every element of the range of $f$ ) exactly once. In particular, if $B$ is a Latin square, $\Gamma_{f, g}$ is $q$-regular.

- If $\Gamma_{f, g}$ is $q$-regular and $A$ is a Latin square, then $B$ is a Latin square.

- If $B$ is a Latin square and no $2 \times 2$ submatrix of $A$ is equal to a $2 \times 2$ submatrix of $B$, then $\Gamma_{f, g}$ is both $q$-regular and contains no 4-cycles.

These observations may provide a basis for a search of new projective planes without translations.

\section{ACKNOWLEDGMENTS}

The authors are very thankful to Gary Ebert for helpful discussions on the subject of finite projective planes, to Gordon Royle for suggesting reference [19], to Rudi Mathon for suggesting references [5], [14], and to an anonymous referee who brought to our attention references [4] and [2]. 


\section{REFERENCES}

[1] B. Bollobás, Extremal Graph Theory, Academic Press, London, 1978.

[2] R. Coulter, R.W. Matthews, Planar Functions and Planes of Lenz-Barlotti Class II, Designs, Codes and Cryptography 10 (1997), 167-184. MR 97j:51010

[3] P. Dembowski, Finite Geometries, Springer-Verlag, Berlin, 1968. MR 38:1597]

[4] P. Dembowski and T.G. Ostrom, Planes of Order $n$ with Collineation Groups of Order $n^{2}$, Math. Zeitschr. 103 (1968), 239-258. MR 37:2075

[5] M.J. de Resmini, On the Mathon plane, J. Geometry 60 (1997) 47-64. MR 99e:51006

[6] A.B. Evans, Maximal sets of mutually orthogonal Latin squares, II, Europ. J. Combinatorics 13 (1992), 345-350. MR 93i:05030

[7] Anthony B. Evans, Orthomorphism graphs of groups. Lecture Notes in Mathematics 1535, Springer-Verlag, Berlin (1992), viii+114 pp. MR 94e:05004

[8] Anthony B. Evans and Robert L. McFarland, Planes of prime order with translations, in Proceedings of the Fifteenth Southeastern Conference on Combinatorics, Graph Theory and Computing, Congr. Numer. 44 (1984) 41-46. MR 86d:51007

[9] David Gluck, A Note on Permutation Polynomials and Finite Geometries, Discrete Mathematics 80 (1990), 97-100. MR 91b:11141

[10] Handbook on Incidence Geometry: buildings and foundations, Edited by F. Buekenhout, Elsevier, North-Holland, 1995. MR 96e:51002

[11] Yutaka Hiramine, A Conjecture on Affine Planes of Prime Order, J. Comb. Theory, Series A 52 (1989), 44-50. MR 90g:51011

[12] D.R. Hughes and F.C. Piper, Projective Planes, Springer-Verlag, New York, 1973. MR 48:12278

[13] F. Lazebnik and A.J. Woldar, General Properties of Some Families of Graphs Defined by Systems of Equations, J. Graph Theory 38 (2001), 65-86. MR 2002k:05108

[14] R. Mathon, On a new projective plane of order 16, Second International Conference in Deinze, 1992. Unpublished talk.

[15] B. McKay, The nauty page, http://cs.anu.edu.au/people/bdm/nauty

[16] L.J. Paige, Ph.D. Dissertation, University of Wisconsin, 1947.

[17] L.J. Paige, A note on finite abelian groups, Bull. Amer. Math. Soc. 53 (1947), 590-593. MR 9:6d

[18] L. Rónyai and T. Szőnyi, Planar Functions Over Finite Fields, Combinatorica 9 (3) (1989), 315-320. MR 91d:51008

[19] T.G. Room and P.B. Kirkpatrick, Miniquaternion Geometry. Cambridge University Press, Cambridge, 1971. MR 45:7590

[20] G. Royle, Projective Planes of Order 16, informal information web-page available at http://www.cs.uwa.edu.au/ gordon/remote/planes 16.

[21] V.A. Ustimenko, personal communication, 1992.

[22] J.H. van Lint and R.M. Wilson, A course in combinatorics, Cambridge University Press, Cambridge (1992), xii+530 pp. MR 94g:05003

[23] O. Veblen and J.H.M. Wedderburn, Non-Desarguesian and non-Pascalian geometries, Trans. Amer. Math. Soc. 8 (1907), 379-388.

[24] Daqing Wan, Gary L. Mullen and Peter Jau-Shyong Shiue, The number of permutation polynomials of the form $f(x)+c x$ over a finite field, Proc. Edinburgh Math. Soc. (2) 38 (1995), 133-149. MR 96e:11161a

Department of Mathematical Sciences, Ewing Building, University of Delaware, Newark, Delaware 19716

E-mail address: lazebnik@math.udel.edu

Department of Pure Mathematics and Mathematical Statistics, Centre for Mathematical Sciences, Wilberforce Road, Cambridge CB3 0WB, United Kingdom

E-mail address: A.G.Thomason@dpmms.cam.ac.uk 